OPEN ACCESS

Edited by:

Giovanni Assenza,

Campus Bio-Medico University, Italy

Reviewed by:

Martina Fanella,

Ospedale San Camillo de Lellis, Italy

Jacopo Lanzone,

Sant'Isidoro Hospital Ferb Onlus

Trescore Balneario, Italy

*Correspondence:

Umberto Aguglia

u.aguglia@unicz.it

Specialty section: This article was submitted to

Epilepsy,

a section of the journa

Frontiers in Neurology

Received: 20 December 2021

Accepted: 25 January 2022

Published: 07 March 2022

Citation:

Mammi A, Ferlazzo E, Gasparini S, Bova V, Neri S, Labate A, Mastroianni G, Bianco CL, Cianci V and Aguglia $U$ (2022) Psychiatric and Behavioural Side Effects Associated

With Perampanel in Patients With

Temporal Lobe Epilepsy. A Real-World Experience. Front. Neurol. 13:839985.

doi: 10.3389/fneur.2022.839985

\section{Psychiatric and Behavioural Side Effects Associated With Perampanel in Patients With Temporal Lobe Epilepsy. A Real-World Experience}

\author{
Anna Mammi ${ }^{1,2}$, Edoardo Ferlazzo ${ }^{1,2}$, Sara Gasparini ${ }^{1,2}$, Valentina Bova ${ }^{2}$, Sabrina Neri ${ }^{1,2}$, \\ Angelo Labate ${ }^{3}$, Giovanni Mastroianni ${ }^{1,2}$, Concetta Lo Bianco ${ }^{1,2}$, Vittoria Cianci ${ }^{2}$ and \\ Umberto Aguglia ${ }^{1,2 *}$
}

${ }^{1}$ Department of Medical and Surgical Sciences, Magna Graecia University, Catanzaro, Italy, ${ }^{2}$ Regional Epilepsy Centre, Great Metropolitan Hospital BMM, Reggio Calabria, Italy, ${ }^{3}$ Neurology Unit, Department of BIOMORF, University of Messina,

Messina, Italy

Psychiatric and behavioural side effects are common, undesirable effects associated with antiseizure medication use. Temporal lobe epilepsy is the most common focal epilepsy in adults and it is frequently associated with drug resistance. Patients with intractable epilepsy are more likely to have psychiatric and behavioural side effects when taking antiseizure medications and seem to be at higher risk for psychiatric comorbidities. Perampanel is a novel anti-seizure medication approved for focal and generalised epilepsies as add-on therapy. This is a 12-week short-term observational prospective study on people with focal epilepsy consecutively recruited from an Italian tertiary epilepsy centre, aimed to compare incidence and severity of psychiatric and behavioural side effects associated with perampanel use in patients with temporal lobe epilepsy as compared to other focal epilepsies. All patients received add-on perampanel according to indication and clinical judgement. Incidence and severity of psychiatric and behavioural side effects were rated by Neuropsychiatric Inventory Questionnaire. All patients enrolled answered the questionnaire before starting perampanel and after 12 weeks of treatment. We found no significant difference in terms of incidence and severity of psychiatric and behavioural side effects associated with perampanel in patients with temporal lobe epilepsy as compared to other focal epilepsies. In line with the literature, the most common adverse effects were "irritability" for both groups and "aggression" for patients with other focal epilepsies.

Keywords: adverse effects, focal epilepsy, psychosis, irritability, anti-seizure medications

\section{INTRODUCTION}

Psychiatric disturbances are more common in people with epilepsy than in the general population (1). People with epilepsy (PWE) may experience psychiatric and behavioural side effects (PBSEs) related to antiseizure medications (ASMSs), including depressed mood, anxiety, psychosis, obsession, personality traits, irritability, aggressive behaviour, and suicide $(2,3)$.

People with drug-resistant epilepsy (seizures failing to disappear with two or more ASMs) are more likely to have PBSEs when taking ASMs (4). Furthermore, pre-existing psychiatric history is a strong predictor of PBSEs in adult patients with epilepsy (5). 
Temporal lobe epilepsy (TLE) is the most common type of focal epilepsy and it is often associated with drug resistance (6). In relation to psychiatric disturbances, a meta-analysis demonstrated that the estimated prevalence of psychosis was higher among individuals with TLE, implicating that the temporal lobe area may be a key brain region, sharing the pathophysiology of TLE and psychiatric disorders (7). Perampanel (PER) is licenced as an add-on treatment for focal onset seizures starting from age 4 and for generalisedonset tonic-clonic seizures in idiopathic generalised epilepsies in people aged 7 years and older (8). PER is a selective, non-competitive antagonist of $\alpha$-amino-3-hydroxy-5-methyl4-isoxazolepropionic acid (AMPA)-type glutamate receptors on post-synaptic neurons and it is the first-in-class AMPA receptor antagonist which acts by reducing glutamatergic transmission (9-11).

Perampanel (PER) treatment is generally well tolerated, with dizziness, somnolence, headache, and fatigue being the most common treatment-emergent side effects (12-14). Pooled data from phase III studies showed elevated rates of PBSEs, in particular hostility/aggression among patients receiving PER compared to those receiving placebo $(15,16)$. It is therefore reasonable to hypothesise that PER may cause a higher incidence of PBSEs in people with TLE, who are more prone to psychiatric comorbidities and ASMs side effects (17). Here we aim to investigate the prevalence and severity of PBSEs associated with PER use as add-on therapy in patients with TLE as compared to other focal epilepsies.

\section{MATERIALS AND METHODS}

The present prospective observational study involved adults with focal epilepsy recruited from the Italian tertiary Centre for epilepsy care (Regional Epilepsy Centre, Grande Ospedale Metropolitano "Bianchi-Melacrino-Morelli", Reggio Calabria, Italy). Inclusion criteria were the following: (a) age > 18 years; (b) inadequate response to one or more ASMs; (c) diagnosis of focal epilepsy according to International League Against Epilepsy (ILAE) $(18,19)$; (d) no change in the ASMs (neither ASM introduction nor changes of dose of pre-existent ASM) during the previous 30 days. Exclusion criteria were: (a) generalised epilepsy; (b) past or current history of alcohol or drug abuse; (c) contraindications to PER use. All recruited participants were started on a once-daily add-on PER at bedtime. An initial dose of $2 \mathrm{mg} /$ day was administered and subsequently increased by $2 \mathrm{mg}$ every $2 / 4$ weeks based on clinical judgement up to a maximum dose of $12 \mathrm{mg} /$ day.

\section{Procedure}

The following data have been collected at baseline for each patient: age, gender, epilepsy aetiology, epileptic syndrome, pre-existing psychiatric comorbidities (i.e., depression, anxiety, personality disorder, psychosis, and psychogenic non-epileptic seizures), ASMs used prior to PER, concomitant ASMs, and duration of PER treatment. The diagnosis of focal epilepsy was made based on clinical, EEG, and MRI criteria (18). Patients were divided into two groups: group 1 (TLE) and group 2 (other focal epilepsies). Lobar localisation was established according to the combination of seizure semiology, ictal/interictal EEG findings, and imaging results based on ILAE classification (19). Incidence and severity of PBSEs in both groups were rated with Neuropsychiatric Inventory-Q (NPI-Q; see below) (20) that was administered to the participants' caregivers before starting PER and after 12 weeks of treatment. PBSEs were searched in all subjects who received at least one dosage of PER and had at least one follow-up clinical evaluation. PBSEs were considered as associated with PER if they were absent before PER or were already present before PER but clearly worsened (score after PER $\geq 1$ ) after PER introduction. Suicidality was also evaluated at each visit. All patients that received PER were included in the analysis.

\section{NPI-Q}

Incidence and severity of PBSEs were assessed through NPI$\mathrm{Q}$ scores at baseline and follow-up times. NPI-Q is a Brief Clinical Form of the Neuropsychiatric Inventory, covering 12 neuropsychiatric symptom domains: depressed mood, euphoria, anxiety, apathy, irritability, aberrant motor behaviours, disinhibition, night-time behavioural disturbances, delusions, hallucinations, appetite/eating disturbances, and aggression.

Furthermore, twelve-symptom domains are assessed by a written screening question that includes core symptom manifestations. Patients are asked to circle "yes" or "no" in response to each question, and to rate the symptoms present in the last 12 weeks if the answer is "yes". Severity neuropsychiatric symptoms are assessed on a three-point scale (1-mild, 2-moderate, and 3-severe). The total NPI-Q severity score represents the sum of individual symptoms scores and ranges from 0 to 36 .

\section{Statistical Analysis}

Descriptive data are expressed as mean \pm standard deviation or number (percentage), as appropriate. Fisher's exact test was used to compare proportions for dichotomic variables in both groups. The nonparametric Mann-Whitney-Wilcoxon (MWW) rank-sum test was used to compare the severity of symptoms. A $p$ $<0.05$ was considered statistically significant. All tests were twotailed. Jamovi 2.2.2 (The Jamovi Project, 2021) (21) was used for all statistical procedures.

\section{RESULTS}

\section{Participants}

Demographic and clinical features of all subjects are shown in Table 1. We enrolled 66 participants (37 women; mean age: $46.9 \pm 16.6$ years). Among the participants, 39 (59.1\%) had TLE (21 women; mean age: $50.3 \pm 16.9$ ), whereas 27 (40.9\%) had other focal epilepsies (16 women; mean age: $42.9 \pm 13.6)$, including nine with frontal lobe epilepsy, nine with parietal lobe epilepsy, four with occipital lobe epilepsy, and five with multifocal or unknown localisation. Before PER introduction, 28 subjects $(42.4 \%)$ had psychiatric comorbidities.

At the time of starting PER, participants took an average of two ASMs. All patients were drug-resistant according to Kwan et al. (22). All the patients received a starting dose of PER of 
TABLE 1 | Demographical and clinical features as a function of focal epilepsies.

\begin{tabular}{|c|c|c|c|}
\hline & $\begin{array}{c}\text { Group } 1 \\
\text { (TLE) } \\
(n=39)\end{array}$ & $\begin{array}{c}\text { Group } 2 \\
\text { (Other focal } \\
\text { epilepsies) } \\
(n=27)\end{array}$ & $p$ value \\
\hline$N(\%)$ & $39(59.1)$ & $27(40.9)$ & 0.17 \\
\hline Age, years (mean $\pm S D$ ) & $50.3 \pm 16.9$ & $42.9 \pm 13.6$ & 0.06 \\
\hline Sex M/F, n (\%) & $\begin{array}{c}18 / 21 \\
(46.2 / 53.8)\end{array}$ & $\begin{array}{c}11 / 16 \\
(40.7 / 59.3)\end{array}$ & 0.80 \\
\hline \multicolumn{4}{|l|}{ Previous ASMs } \\
\hline $1-3, \mathrm{n}(\%)$ & $24(61.5)$ & $12(44.4)$ & 0.17 \\
\hline $4-5, \mathrm{n}(\%)$ & $5(12.8)$ & $7(25.9)$ & 0.17 \\
\hline$\geq 6, \mathrm{n}(\%)$ & $10(25.6)$ & 8 (29.6) & 0.72 \\
\hline PER dose, mg/day (mean \pm SD) & $7.2 \pm 2.7$ & $7,1 \pm 2.5$ & 0.81 \\
\hline Concomitant ASMs (mean \pm SD) & $2.1 \pm 0.7$ & $2.1 \pm 0.7$ & 0.96 \\
\hline $1, \mathrm{n}(\%)$ & $8(20.5)$ & $5(18.5)$ & 0.84 \\
\hline $2, \mathrm{n}(\%)$ & $20(51.3)$ & $15(55.6)$ & 0.73 \\
\hline $3, \mathrm{n}(\%)$ & $10(25.6)$ & $6(22.2)$ & 0.75 \\
\hline 4, n (\%) & $1(2.6)$ & $1(3.7)$ & 0.79 \\
\hline Psychiatric disorders (PD) n (\%) & $19(48.7)$ & $9(33.3)$ & 0.31 \\
\hline Depressed mood n (\%) & $14(35.9)$ & $5(18.5)$ & 0.17 \\
\hline Anxiety $n(\%)$ & $4(10.3)$ & $3(11.1)$ & 1 \\
\hline Psychosis n (\%) & $7(17.9)$ & $4(14.8)$ & 1 \\
\hline $\begin{array}{l}\text { Concomitant psychotropic drugs } \\
\text { n (\%) }\end{array}$ & $19(48.7)$ & $9(33.3)$ & 0.31 \\
\hline Antidepressant n (\%) & $14(35.8)$ & $5(19.5)$ & 0.79 \\
\hline Anxiolytic n (\%) & $16(41)$ & $8(29.6)$ & 0.43 \\
\hline Antipsychotic n (\%) & $7(17.9)$ & $4(14.8)$ & 1 \\
\hline
\end{tabular}

$2 \mathrm{mg} /$ day, with an increase of $2 \mathrm{mg} /$ day every 2-4 weeks when indicated, up to a maximum dose of $12 \mathrm{mg} /$ day.

At the time of follow-up, after 12 weeks, 59 (89.4\%) patients were still taking PER, whereas seven patients $(10.6 \%)$ discontinued PER, two (3\%) because of PBSEs, five $(7.6 \%)$ because of other adverse effects (dizziness, somnolence, and asthenia). Among the patients who discontinued PER, 6/7 had TLE and $1 / 7$ extra-TLE $(p=0.23)$. PER median dose after 12 week-treatment was $7.2 \pm 2.6 \mathrm{mg} /$ day for all patients. No significant differences were found between groups 1 and 2 in terms of demographic variables.

\section{Incidence and Severity of PBSEs}

Incidence and severity of PBSEs related to PER were rated by NPI-Q patients scores signed at baseline and follow-up.

Considering the whole sample, $13 / 66$ patients $(19.7 \%)$ experienced from 1 to 3 PBSEs, 13/66 (19.7\%) experienced "irritability", 2/66 (3\%) experienced "aggression", 1/66 (1.5\%) had "depression", 1/66 (1.5\%) had "aberrant motor behaviours", and 1/66 (1.5\%) had "night-time behavioural disturbances".

In group $1,8 / 39(20.5 \%)$ subjects experienced from 1 to 3 PBSEs, $1 / 39$ (2.6\%) experienced “depression", 8/39 patients (20.5\%) experienced "irritability", 1/39 (2.6\%) had "aberrant motor behaviours", and 1/39 (2.6\%) had "night-time behavioural
TABLE 2 | Incidence and severity of BPSEs as functions of focal epilepsies.

\begin{tabular}{lccc}
\hline & $\begin{array}{c}\text { Group 1 (TLE) } \\
(\boldsymbol{n}=\mathbf{3 9})\end{array}$ & $\begin{array}{c}\text { Group 2 (Other } \\
\text { focal } \\
\text { epilepsies) } \\
(\boldsymbol{n}=\mathbf{2 7})\end{array}$ & $\begin{array}{c}\boldsymbol{p} \\
\text { value }\end{array}$ \\
\hline $\begin{array}{l}\text { Patients experienced } \\
\text { PBSEs n (\%) }\end{array}$ & $8(20.5)$ & $5(18.5)$ & 0.95 \\
$\begin{array}{l}\text { The severity of symptoms } \\
\text { median (IQR) }\end{array}$ & $5.5(\mathrm{IQR}, 3-7.25)$ & $5(\mathrm{IQR}, 3-7)$ & 0.82 \\
$\begin{array}{l}\text { Depressed mood n (\%) } \\
\text { Irritability n (\%) }\end{array}$ & $1(2.6)$ & 0 & 0.40 \\
$\begin{array}{l}\text { Aberrant motor behaviours } \\
\mathrm{n} \text { (\%) }\end{array}$ & $8(20.5)$ & $5(18.5)$ & 0.84 \\
$\begin{array}{l}\text { Night-time behavioural } \\
\text { disturbances n (\%) }\end{array}$ & $1(2.6)$ & 0 & 0.40 \\
Aggression n (\%) & $1(2.6)$ & 0 & 0.40 \\
\hline
\end{tabular}

disturbances". The median severity score was 5.5 (IQR, 3-7,25). In group 2, 5 subjects experienced PBSEs, all patients (18.5\%) had "irritability" with two of them experiencing aggression too. No difference was found between the two groups (for more details see Table 2). Suicide attempts were not experienced by any patients.

\section{DISCUSSION}

In the present study, we found no significant difference in terms of incidence and severity of psychiatric and behavioural side effects associated with PER in people with TLE as compared to other focal epilepsies. PBSEs appeared in $19.7 \%$ of patients, in agreement with literature data, without significant difference between people with TLE (20.5\%) and other focal epilepsies (18.5\%). Indeed, double-blind randomised controlled trial (RCT) studies showed that PER is generally well tolerated with the most common adverse events being dizziness, somnolence, headache, fatigue, irritability, and aggression in about $20 \%$ of patients (12-14). Moreover, several real-world studies showed a similar rate of PBSEs at 1 to 3-year follow-up (23-27). Mild to moderate PBSEs (mainly irritability, anxiety, verbal aggressivity, agitation/restlessness, depression, psychosis, and suicidal ideation) were reported in about $15-25 \%$ of patients. Furthermore, people with a history of psychiatric comorbidities showed a significantly higher risk of developing psychosis and suicidal ideation.

To our knowledge, no other study comparing the incidence and severity of PBSEs associated with PER use in PWE and different lobar localisation has been conducted so far. Likewise, there aren't any studies exploring the incidence of PBSEs associated with other ASMs in different types of focal epilepsies, although it is reported that PBSEs are more likely to occur in people with psychiatric comorbidity and who are considered to be at risk of psychiatric disorders, like TLE people (28).

In our study, we found no higher incidence of PBSEs in people with TLE. We might hypothesise that concomitant ASM and other psychotropic medications are taken may have mitigated psychiatric adverse side effects. However, we found no difference 
in terms of concomitant medications in our two groups and this might be explained by the small sample size of the present study.

Moreover, people with TLE may have an increased risk of developing psychiatric comorbidity without a higher risk to experience PBSEs associated with ASMs because of the different pathogenic mechanisms underlying both psychiatric symptoms.

It is well known that a strong and bidirectional relationship between epilepsy and psychiatric disturbance. Hence, it could be hypothesised a common etiopathology of neurological and psychiatric disorders based on both genetic and environmental factors. On the other hand, PBSEs associated with ASMs are pharmacologically induced phenomena and are strictly dependent on ASM mechanisms of action, interactions with other psychotropic medications, pre-existent psychiatric disturbances, personality and life quality of PWE, change in seizure frequency (force normalisation), and other personal risk and protective factors of psychiatric symptoms.

Our study has some limitations. It is an observational study in which participants are not randomised and a control group of patients treated with other ASMs is not included. Furthermore, the sample size is limited and follow-up time ( 12 weeks) may be too short to exclude the occurrence of PBSEs. So, our findings require further validation from studies with larger sample sizes and longer follow-up.

\section{REFERENCES}

1. Kanner, AM. Management of psychiatric and neurological comorbidities in epilepsy. Nat Rev Neurol. 12:106-16. doi: 10.1038/nrneurol.2015.243

2. Weintraub D, Buchsbaum R, Resor SR Jr, Hirsch LJ. Psychiatric and behavioral side effects of the newer antiepileptic drugs in adults with epilepsy. Epilepsy Behav. (2007) 10.1:105-10. doi: 10.1016/j.yebeh.2006.08.008

3. Schmitz B. Psychiatric syndromes related to antiepileptic drugs. Epilepsia. (1999) 40:S65-70. doi: 10.1111/j.1528-1157.1999.tb00887.x

4. Chen B, Choi H, Hirsch LJ, Katz A, Legge A, Buchsbaum R, et al. Psychiatric and behavioral side effects of antiepileptic drugs in adults with epilepsy. Epilepsy Behav. (2017) 76:24-31. doi: 10.1016/j.yebeh.2017. 08.039

5. Trimble MR, Rüsch N, Betts T, Crawford PM. Psychiatric symptoms after therapy with new antiepileptic drugs: psychopathological and seizure related variables. Seizure. (2000) 9.4:249-54. doi: 10.1053/seiz.2000.0405

6. Engel J, McDermott MP, Wiebe S, Langfitt JT, Stern JM, Dewar S. Early, Randomized Surgical Epilepsy Trial (ERSET) Study Group. Early surgical therapy for drug-resistant temporal lobe epilepsy: a randomized trial. JAMA. (2012). 307:922-930. doi: 10.1001/jama.2012.220

7. Clancy MJ, Clarke MC, Connor DJ, Cannon M, Cotter DR. The prevalence of psychosis in epilepsy; a systematic review and meta-analysis. BMC Psychiatry. (2014) 13:14-75. doi: 10.1186/1471-244X-14-75

8. European Medicine Agency (2021). Fycompa: EPAR - Product Information. Available online at: https://www.ema.europa.eu. (accessed September 12, 2021).

9. Hanada T, Hashizume Y, Tokuhara N, Takenaka O, Kohmura N, Ogasawara A, et al. Perampanel: a novel, orally active, noncompetitive AMPA-receptor antagonist that reduces seizure activity in rodent models of epilepsy. Epilepsia. (2011) 52:1331-40. doi: 10.1111/j.1528-1167.2011.03109.x

10. Rogawski MA, Hanada T. Preclinical pharmacology of perampanel, a selective non-competitive AMPA receptor antagonist. Acta Neurol Scand Suppl. (2013) 197:19-24. doi: 10.1111/ane.12100

11. Di Bonaventura C et al. "AMPA receptors and perampanel behind selected epilepsies: current evidence and future perspectives. Expert Opin Pharmacother. (2017) 18:1751-64. doi: 10.1080/14656566.2017.1392509

\section{DATA AVAILABILITY STATEMENT}

The original contributions presented in the study are included in the article/supplementary material, further inquiries can be directed to the corresponding author.

\section{ETHICS STATEMENT}

Ethical review and approval was not required for the study on human participants in accordance with the local legislation and institutional requirements. Written informed consent for participation was not required for this study in accordance with the national legislation and the institutional requirements.

\section{AUTHOR CONTRIBUTIONS}

$\mathrm{VC}$ and $\mathrm{AL}$ contributed to conception and design of the study. GM, VB, and $\mathrm{SN}$ organised the database. $A M$ and $C B$ wrote the first draft of the manuscript. $\mathrm{EF}$ and SG wrote sections of the manuscript. UA contributed to manuscript revision. All authors contributed to manuscript revision, read, and approved the submitted version.

12. French JA, Krauss GL, Biton V, Squillacote D, Yang H, Laurenza $A$, et al. Adjunctive perampanel for refractory partial-onset seizures: randomized phase III study 304. Neurology. (2012) 79:589-596. doi: 10.1212/WNL.0b013e3182635735

13. French JA, Krauss GL, Steinhoff BJ, Squillacote D, Yang H, Kumar D, et al. Evaluation of adjunctive perampanel in patients with refractory partial-onset seizures: results of randomized global phase III study 305. Epilepsia. (2013) 54:117-25. doi: 10.1111/j.1528-1167.2012.03638.x

14. Krauss GL, Serratosa JM, Villanueva V, Endziniene M, Hong Z, French J, et al. Randomized phase III study 306: adjunctive perampanel for refractory partial-onset seizures. Neurology. (2012) 78:1408-15. doi: 10.1212/WNL.0b013e318254473a

15. Steinhoff BJ, Ben-Menachem E, Ryvlin P, Shorvon S, Kramer L, Satlin A, et al. Efficacy and safety of adjunctive perampanel for the treatment of refractory partial seizures: a pooled analysis of three phase III studies. Epilepsia. (2013) 54:1481-9. doi: 10.1111/epi.12212

16. Maurousset A, Limousin N, Praline J, Biberon J, Corcia P, De Toffol B. Adjunctive perampanel in refractory epilepsy: Experience at tertiary epilepsy care center in Tours. Epilepsy Behav. (2016) 61:237-41. doi: 10.1016/j.yebeh.2016.06.005

17. Vinti Vet al. "Temporal lobe epilepsy and psychiatric comorbidity." Frontiers in Neurology: 2155.

18. Scheffer IE, Berkovic S, Capovilla G, Connolly MB, French J, Guilhoto L, et al. ILAE classification of the epilepsies: Position paper of the ILAE commission for classification and terminology. Epilepsia. (2017) 58:51221. doi: 10.1111/epi.13709

19. Fisher RS, Cross JH, French JA, Higurashi N, Hirsch E, Jansen FE, et al. Operational classification of seizure types by the International League Against Epilepsy: Position Paper of the ILAE Commission for Classification and Terminology. Epilepsia. (2017) 58:522-30. doi: 10.1111/epi.13670

20. Kaufer DI, Cummings JL, Ketchel P, Smith V, MacMillan A, Shelley $\mathrm{T}$, et al. Validation of the NPI-Q, a brief clinical form of the Neuropsychiatric Inventory. J Neuropsychiatry Clin Neurosci. (2000) 12:233-9. doi: 10.1176/jnp.12.2.233

21. The jamovi project (2021). Jamovi (Version 2.2.2). Available online at: https:// www.jamovi.org/ (accessed September 12, 2021). 
22. Kwan P, Arzimanoglou A, Berg AT, Brodie MJ, Allen Hauser W, Mathern G, et al. Definition of drug resistant epilepsy: consensus proposal by the ad hoc task force of the ILAE commission on therapeutic strategies. Epilepsia. (2010) 51:1069-77. doi: 10.1111/j.1528-1167.2009.02397.x

23. Morano A, Fattouch J, Albini M, Casciato S, Fanella M, Basili LM, et al. Perampanel as adjunctive therapy in highly refractory epilepsies: Real-world data from an Italian tertiary care epilepsy centre. J Neurol Sci. (2018) 390:6774. doi: 10.1016/j.jns.2018.04.017

24. Villanueva V, Garcés M, López-González FJ, Rodriguez-Osorio X, Toledo M, Salas-Puig J, et al. Safety, efficacy and outcome-related factors of perampanel over 12 months in a real-world setting: The FYDATA study. Epilepsy Res. (2016) 126:201-10. doi: 10.1016/j.eplepsyres.2016. 08.001

25. Trinka E, Steinhoff BJ, Nikanorova M, Brodie MJ. Perampanel for focal epilepsy: insights from early clinical experience. Acta Neurol Scand. (2016) 133:160-72. doi: 10.1111/ane.12529

26. Labate A, Fortunato F, Giugno A, Martino I, Caligiuri ME, Gambardella A, et al. Perampanel as first add-on choice on the treatment of mesial temporal lobe epilepsy: an observational real-life study. Neurol Sci. (2021) 442:138994. doi: 10.1007/s10072-020-04636-7

27. Pascarella A, et al. The efficacy of perampanel as adjunctive therapy in drugresistant focal epilepsy in a "real world" context: focus on temporal lobe epilepsy. J Neurol Sci. (2020) 415:116903. doi: 10.1016/j.jns.2020.116903
28. Ettinger AB, LoPresti A, Yang H, Williams B, Zhou S, Fain R, et al. Psychiatric and behavioral adverse events in randomized clinical studies of the noncompetitive AMPA receptor antagonist perampanel. Epilepsia. (2015) 56:1252-63. doi: 10.1111/epi.13054

Conflict of Interest: The authors declare that the research was conducted in the absence of any commercial or financial relationships that could be construed as a potential conflict of interest.

Publisher's Note: All claims expressed in this article are solely those of the authors and do not necessarily represent those of their affiliated organizations, or those of the publisher, the editors and the reviewers. Any product that may be evaluated in this article, or claim that may be made by its manufacturer, is not guaranteed or endorsed by the publisher.

Copyright (C) 2022 Mammi, Ferlazzo, Gasparini, Bova, Neri, Labate, Mastroianni, Bianco, Cianci and Aguglia. This is an open-access article distributed under the terms of the Creative Commons Attribution License (CC BY). The use, distribution or reproduction in other forums is permitted, provided the original author(s) and the copyright owner(s) are credited and that the original publication in this journal is cited, in accordance with accepted academic practice. No use, distribution or reproduction is permitted which does not comply with these terms. 